\title{
Parallel Ion Electron Spectrometry (PIES): A New Paradigm for High- Resolution High-Sensitivity Characterization based on integrated TEM-SIMS
}

\author{
S. Eswara Moorthy, D. Dowsett, L. Yedra, T. Wirtz
}

Advanced Instrumentation for Ion Nano-Analytics (AINA), Luxembourg Institute of Science and Technology, 41 rue du Brill, L-4422 Belvaux, Luxembourg

The Transmission Electron Microscopy (TEM) offers superior spatial resolution, but, the traditional analytical capabilities associated with electron microscopy such as the Energy Dispersive Spectroscopy (EDS) or Electron Energy-Loss Spectroscopy (EELS) are unfortunately inadequate for characterizing samples containing trace elements (at best 0.1 at. \%) or for mapping isotopic distributions [1,2]. Another limitation is that investigations of light elements (such as hydrogen and lithium) are particular difficult or even impossible using these analytical methods. On the other hand, Secondary Ion Mass Spectrometry (SIMS) provides extraordinary chemical sensitivity (down to ppm or even ppb) and high dynamic range, but, offers poor lateral resolution [3]. However, to tackle modern problems in physical and biological sciences, the capability to simultaneously obtain high spatial resolution and high chemical sensitivity is of paramount importance. An ex-situ combination of TEM and SIMS in an attempt to overcome the limitations of the techniques taken individually is prone to sample modifications and other artefacts [4]. To overcome the intrinsic instrumental limitations, we have made an in-situ combination to complement the high-sensitivity of SIMS with the exceptional spatial resolution offered by TEM, by developing the correlative TEMSIMS technique.

To determine the feasibility and to demonstrate the applications of the TEM-SIMS technique, we have developed a prototype instrument for TEM-SIMS based correlative microscopy (Figure 1). The pole-pieces of a Tecnai F20 were specially modified to accommodate the SIMS technique. A commercial FEI Magnum $\mathrm{Ga}^{+}$FIB was attached to the TEM column to act as the primary ion column. The secondary ion extraction optics (extraction efficiency 90\%) and a compact highperformance mass spectrometer were designed and developed in-house and are being continuously improved for optimal performance. A special sample holder which can be biased to high-voltages $( \pm 4.5 \mathrm{kV})$ was also developed in-house to enhance the collection efficiency of the secondary-ion extraction optics.

To enhance the low intrinsic yield of secondary ions for non-reactive primary ion beams such as $\mathrm{Ga}^{+}$ for the TEM-SIMS we use reactive gas flooding [5]. Specifically, the enhancement of negative secondary ion yields due to Cs flooding and of positive secondary ion yields with $\mathrm{O}_{2}$ flooding were found to be up to four orders-of-magnitude. This enhancement of secondary ion yields leads to detection limits varying from $10^{-3}$ to $10^{-6}$ for a lateral resolution between $10 \mathrm{~nm}$ and $100 \mathrm{~nm}$ respectively (Figure 2). Sensitivities in the ppm range are possible, but at the cost of spatial resolution due to the inherent physical limit of SIMS. Nevertheless, it is possible to recover the structural details that were thus lost by overlaying high-resolution TEM image over the highsensitivity (but, poorer resolution) SIMS image.

In this presentation, the strategies employed to overcome the technical challenges of the TEM-SIMS combination of techniques will be discussed and the new possibilities enabled by this correlative microscopy method will be highlighted with a focus on applications in materials science and 
biology. Two distinctive analysis protocols were identified: (a) TEM is used first to identify interesting structures followed by SIMS to know the chemical information and (b) SIMS is used first to identify chemical hotspots and then TEM is used for structural characterization. Details of these protocols and other related methods like isotopic labelling will also be discussed. Special emphasis will also be placed on TEM-SIMS characterization of samples containing light elements (low Z), which are particularly challenging with traditional analytical methods like EDS or EELS.

References:

[1] L. Reimer, H. Kohl, Transmission Electron Microscopy: physics of image formation (Springer, New York, 2008).

[2] R. F. Egerton, Electron Energy-Loss Spectroscopy in the Electron Microscope (3rd edition), (Springer, New York, 2011).

[3] D. S. McPhail, J. Mater. Sci. 41, (2006) 873.

[4] K. Q. Ngo et al, Surf. Sci. 606, (2012) 1244.

[5] P. Philipp et al, Int. J. Mass. Spectrom. 253 (2006) 71

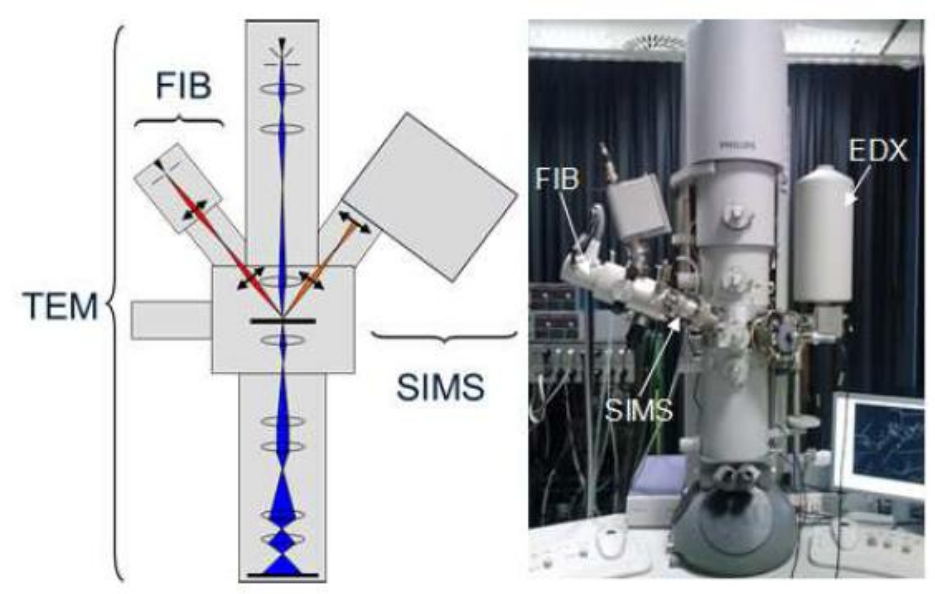

Figure 1. Schematic of the TEM-SIMS setup (left) and the photo of the TEM-SIMS prototype instrument (right).
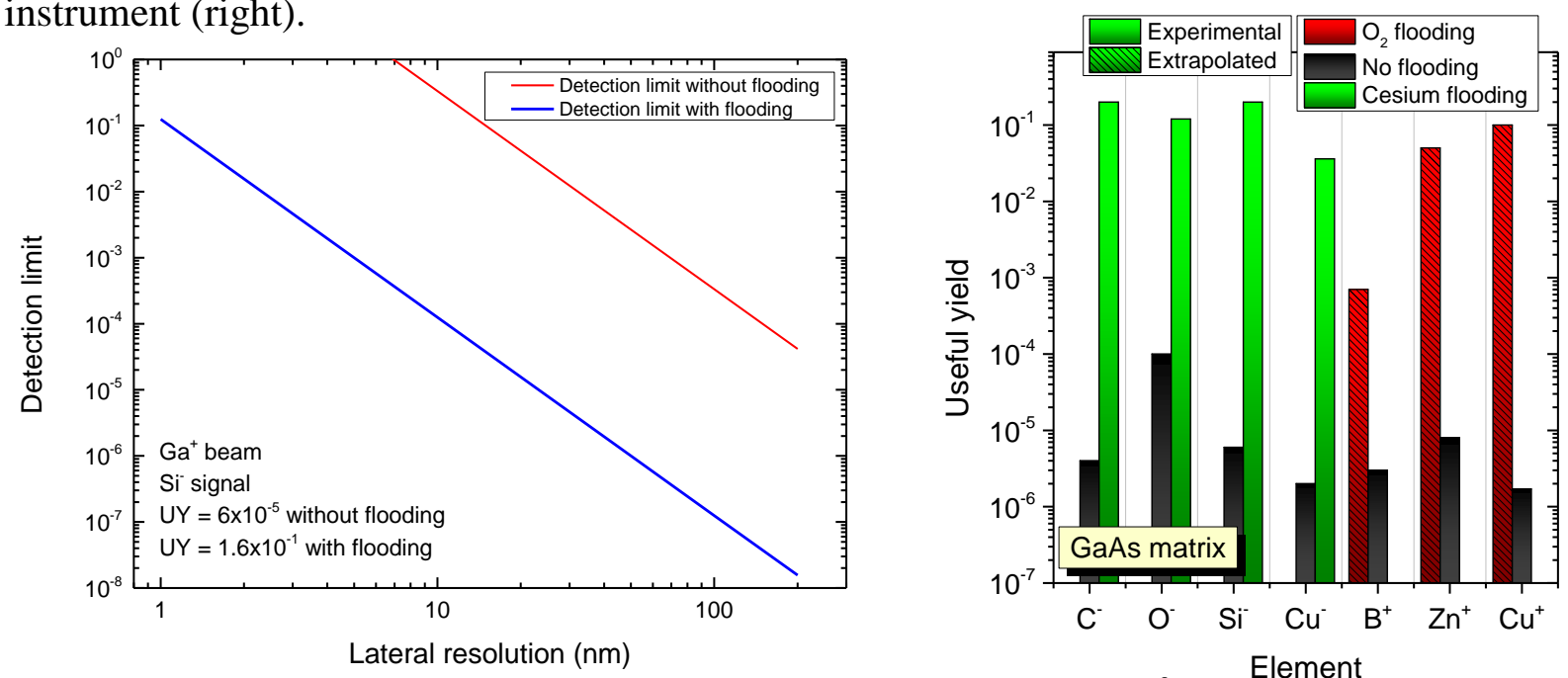

Figure 2. Left: Detection limits using a $\mathrm{Ga}^{+} \mathrm{FIB}$ with and without $\mathrm{Cs}^{0}$ flooding vs. minimum feature size: example for the detection of $\mathrm{Si}^{-}$. Right: Enhancement of secondary ion yields using reactive gas flooding under $\mathrm{Ga}^{+}$bombardment 